\title{
PLESIOMONAS SHIGELLOIDES AND AEROMONADACEAE FAMILY PATHOGENS ISOLATED FROM MARINE MAMMALS OF SOUTHERN AND SOUTHEASTERN BRAZILIAN COAST
}

\author{
Christiane S. Pereira ${ }^{1 *}$; Simone D. Amorim ${ }^{1}$; André Felipe das M. Santos ${ }^{1}$; Salvatore Siciliano²; \\ Ignacio B. Moreno ${ }^{3,4}$; Paulo Henrique Ott ${ }^{3,4}$; Dalia dos Prazeres Rodrigues ${ }^{1}$ \\ ${ }^{1}$ Laboratório de Referência Nacional de Cólera e outras Enteroinfecções Bacterianas, Instituto Oswaldo Cruz, \\ Fundação Oswaldo Cruz, Manguinhos, RJ, Brasil; ${ }^{2}$ Laboratório de Endemias, Escola Nacional de Saúde Pública, \\ Fundação Oswaldo Cruz, Manguinhos, RJ, Brasil; ${ }^{3}$ Grupo de Estudos de Mamíferos Aquáticos do Rio Grande do Sul, \\ Porto Alegre, RS, Brasil; ${ }^{4}$ Centro de Estudos Costeiros, Limnológicos e Marinhos, Universidade Federal do Rio Grande \\ do Sul, Imbé, RS, Brasil
}

Submitted: January 02, 2008; Returned to authors for corrections: March 05, 2008; Approved: November $02,2008$.

\begin{abstract}
The aquatic environment is the habitat of many microorganisms, including Plesiomonas shigelloides and Aeromonas species which are pathogenic to human and animals. In the present investigation, we evaluated the occurrence of these pathogens from marine mammals beached or accidentally captured by fishing net in southeastern (RJ) and southern (RS) coastal Brazilian regions. A total of 198 swabs from 27 specimens of marine mammals, including 11 different species, were collected by DEENSP and GEMARS-CECLIMAR/ UFRGS Institutes and sent to LRNCEB/IOC/FIOCRUZ. The samples were enriched in Alkaline Peptone Water (APW) added with $1 \%$ of sodium chloride $(\mathrm{NaCl})$, APW plus $3 \% \mathrm{NaCl}$ and incubated at $37^{\circ} \mathrm{C}$ for $18-24$ hours. Following, samples were streaked onto Pseudomonas-Aeromonas Selective Agar Base (GSP Agar) and suspected colonies were biochemically characterized. The results revealed 114 strains, including ten Aeromonas species and $P$. shigelloides. The main pathogens isolated were $A$. veronii biogroup veronii (19.3\%), A. caviae $(12.3 \%), A$. hydrophila $(9.6 \%)$ and $P$. shigelloides $(7 \%)$. The pathogens were isolated in both coastal and offshore marine mammals. These data point the importance of epidemiological surveillance and microbiological monitoring and reinforce the need to implement environmental protection programs, especially related to endangered cetacean species.
\end{abstract}

Key words: Aeromonas, Plesiomonas shigelloides, marine mammals, aquatic ecosystem.

\section{INTRODUCTION}

Marine ecosystem is recognized as a natural habitat of some pathogenic microorganisms. The infectious diseases caused by these pathogens are dangerous to marine animal's health and can potentially affect several species, including endangered marine mammals. Nevertheless, the causative agents of the diseases and their risks to animal and human health are difficult to determine, especially due to the migratory habits of some marine mammals species $(4,23)$.
Marine mammals are probably the best sentinel organisms in aquatic and coastal environments, because many species have long life spans and are at the top of food chain $(2,25)$. Therefore, some pathogens (e.g. bacteria and virus) isolated from these animals could be used as indicators of disturbance in the marine ecosystem.

Global warming as well as the El Niño phenomenon can have an important effect on the marine food web and, consequently, on the dynamic and health of marine mammal populations $(17,19,26,29)$. In addition, the increasing degradation of the marine

*Corresponding Author. Mailing address: Laboratório de Referência Nacional de Cólera e outras Enteroinfecções Bacterianas (LRNCEB) Instituto Oswaldo Cruz (IOC) Fundação Oswaldo Cruz (FIOCRUZ). Avenida Brasil, 4365, Pavilhão Rocha Lima 3o andar sala 320. CEP 21045-900. Manguinhos, Rio de Janeiro. E-mail: chrisspm@ioc.fiocruz.br 
ecosystem have been led to a decline of fisheries resources and exposed marine mammals to a variety of pollutants and toxic substances that can negatively affect their survival (12).

These ecological and climatic changes in the marine environment contribute to the occurrence of new and reemergent diseases affecting marine mammals (2). The Aeromonadaceae family is a group of microorganisms spread in aquatic ecosystems and much of them are pathogenic to animals and humans. These bacteria are associated to a wide range of skin disorders in fishes, including one of the major diseases in aquaculture (furunculosis). In humans, Aeromonas have been increasingly recognized as a relevant etiological agent in gastrointestinal infections, possibly associated to the ingestion of contaminated water or food products $(7,8,10)$.

Plesiomonas shigelloides is an ubiquitous bacterium, widely distributed in fresh and estuarine water. This microorganism is as considered an opportunistic pathogen isolated from normal bacterial flora of healthy animals and also from captive marine mammals. Both Aeromonas and Plesiomonas may cause systemic or local lesions on skin and fins of marine mammals, especially when these animals are submitted to stress conditions. Furthermore, immunocompromised people are more susceptible to infections caused by these pathogens $(11,20)$.

In Brazil, several studies pointed out the occurrence of these pathogens in the aquatic ecosystem, as well as in seafood products (fishes, oysters, mussels and crabs) and in cutaneous lesions of fishermen. These studies are important to Public Health due to the association between some of these microorganisms and the incidence of foodborne disease and extraintestinal infections $(13,22,24)$.

The aim of this study was to evaluate the occurrence of the genus Aeromonas and $P$. shigelloides from superficial lesions (wounds) and other sites (e.g. mouth, blowhole, genital slit and anus) of marine mammals beached or accidentally captured by fishing net in Rio de Janeiro and Rio Grande do Sul, Brazil.

\section{MATERIALS AND METHODS}

In the present investigation we evaluated a total of 198 swabs (Cary-Blair transport medium) from 27 marine mammal specimens, including five dolphin species: franciscana (Pontoporia blainvillei) $(\mathrm{n}=12)$, marine tucuxi dolphin (Sotalia guianensis) $(\mathrm{n}=3)$, Atlantic spotted dolphin (Stenella frontalis) $(\mathrm{n}=3)$, rough-toothed dolphin (Steno bredanensis) $(\mathrm{n}=1)$, common dolphin (Delphinus sp.) $(\mathrm{n}=1)$; one beaked whale: True's beaked whale (Mesoplodon mirus) $(\mathrm{n}=1)$; four baleen whales: southern right whale (Eubalaena australis) $(\mathrm{n}=2)$, Bryde's whale (Balaenoptera edeni) $(\mathrm{n}=1)$, humpback whale (Megaptera novaeangliae) $(\mathrm{n}=1)$, dwarf minke whale (Balaenoptera acutorostrata) $(\mathrm{n}=1)$; and one pinniped species: South American sea lion (Otaria byronia) $(\mathrm{n}=1)$. A summary of data, including geographical area, the total animal number beached or accidentally captured, and positive/negative swabs collected are detailed in Table 1.

From 2003 to 2004, swabs from mouth, eyes, nostrils, umbilicus, genital slit, anus, and open wounds of marine mammals were collected by DEENSP (Departamento de Endemias da Escola Nacional de Saúde Pública Sérgio Arouca FIOCRUZ) in Rio de Janeiro State and by GEMARS/CECLIMAR (Grupo de Estudos de Mamíferos Aquáticos do Rio Grande do Sul/Centro de Estudos Costeiros, Limnológicos e Marinhos da Universidade Federal do Rio Grande do Sul) in Rio Grande do Sul State. These institutes have been conducted long-term research and rehabilitation of marine mammals along the southern

Table 1. Summary of the swabs collected from marine mammals beached or accidentally captured by fishing net in Rio de Janeiro (RJ) and Rio Grande do Sul (RS), Brazil.

\begin{tabular}{|c|c|c|c|c|c|c|}
\hline \multirow{2}{*}{ Marine mammals } & \multirow{2}{*}{ Habitat } & \multirow{2}{*}{ Number of animals } & \multicolumn{3}{|c|}{ Swabs } & \multirow{2}{*}{ Region } \\
\hline & & & Total & Positive & Negative & \\
\hline Pontoporia blainvillei & Coastal & 12 & 114 & 52 & 62 & RS \\
\hline Sotalia guianensis & Coastal & 3 & 19 & 8 & 11 & RJ \\
\hline Stenella frontalis & Coastal/Oceanic & 3 & 9 & $8^{*}$ & $1 * *$ & $\mathrm{RS}^{*}, \mathrm{RJ}^{* *}$ \\
\hline Eubalaena australis & Coastal & 2 & 9 & $3 *$ & $6^{* *}$ & $\mathrm{RJ}^{*}, \mathrm{RS}^{* *}$ \\
\hline Otaria flavescens & Coastal & 1 & 22 & 5 & 17 & RS \\
\hline Balaenoptera edeni & Coastal/Oceanic & 1 & 5 & 0 & 5 & RJ \\
\hline Steno bredanensis & Coastal & 1 & 6 & 1 & 5 & RJ \\
\hline Delphinus sp. & Coastal & 1 & 5 & 4 & 1 & RS \\
\hline Mesoplodon mirus & Oceanic & 1 & 5 & 3 & 2 & RJ \\
\hline Balaenoptera acutorostrata & Coastal & 1 & 3 & 0 & 3 & RJ \\
\hline Megaptera novaeangliae & Coastal & 1 & 1 & 0 & 1 & RS \\
\hline Total & - & 27 & 198 & 84 & 114 & - \\
\hline
\end{tabular}


and southeastern Brazilian coast. Samples were sent to Laboratório de Referência Nacional de Cólera e outras Enteroinfecções Bacterianas (LRNCEB), Instituto Oswaldo Cruz (IOC), Fundação Oswaldo Cruz (FIOCRUZ) in order to evaluate the occurrence of Plesiomonas shigelloides and Aeromonadaceae family pathogens.

Samples were enriched at Alkaline Peptone Water (APW) containing $1 \%$ sodium chloride $(\mathrm{NaCl})$ and $\mathrm{APW}$ plus $3 \% \mathrm{NaCl}$ $\left(37^{\circ} \mathrm{C} / 18-24\right.$ hours). Thereafter, samples were streaked onto Pseudomonas-Aeromonas Selective Agar Base (GSP Agar) and the oxidase positive colonies were submitted to biochemical tests: susceptibility to $\mathrm{O} / 129$ vibriostatic agent $(2,4$ diamino- 6 , 7 diisoprylpteridine), ONPG production ( $\alpha$ nitrophenyl- $\beta-D$ galactopyranoside), VP(Voges-Proskauer), lysine and ornithine descarboxylase and arginine dihydrolase $(1,9)$.

\section{RESULTS AND DISCUSSION}

From the eleven species of marine mammal investigated in this study, only humpback, dwarf minke and Bryde's whales samples were not positive for Aeromonas and Plesiomonas shigelloides. The highest frequency of such microorganisms was detected in three coastal marine mammals (P. blainvillei, $S$. guianensis and $O$. flavescens) (Table 1), although this result can be bias by the higher number of swabs collected from these species.

A total of 114 bacterial strains were identified, including 10 Aeromonas species and $P$. shigelloides. The main pathogens isolated were $A$. veronii biogroup veronii (19.3\%), A caviae (12.3\%), A. hydrophila (9.6\%), A. veronii biogroup sobria (8.8\%), and P. shigelloides (7,0\%). Another Aeromonas species were isolated at lower rates, such as $A$. jandaei (5.3\%), A. sobria (5.3\%), A. schubertii (4.4\%), A. media (4.4\%), A. eucrenophila (4.4\%) and $A$. trota $(3.5 \%)$. In addition, $15.8 \%$ of the strains were identified only until the genus level (Aeromonas). The microorganisms isolated from each marine mammal species are described in Table 2.

Regarding the source of bacterial isolation, a higher diversity of microorganisms were isolated from the blowhole/nostril (11 species), genital slit ( 9 species) and anus ( 9 species) of the marine mammals, with the predominance of $A$. veronii biogroup veronii. On the other hand, the most widespread species were A. veronii biogroup sobria and $P$. shigelloides, which were isolated from the mouth, blowhole/nostril, eye, genital slit and anus of the animals. Aeromonas veronii biogroup sobria was also isolated from a dolphin intestine (Tables 3 and 4).

Considering the widespread distribution of bacteria among marine mammal species, additional studies should be develop to increase the knowledge about their virulence in cases of opportunistic infections, especially the ones caused after stress conditions.

Information about microorganisms, including the ones potentially zoonotic agents for marine mammals isolated from Brazilian waters is almost inexistent (23). However, our observations indicated that there is a relatively large group of bacteria, including potential pathogens, that exist in marine mammals in this area. Two species reported in this study ( $A$. hydrophila and $P$. shigelloides) were also found in free-ranging and presumably healthy bottlenose dolphins (Tursiops truncatus) from Florida, North Carolina (3). Nevertheless,

Table 2. Plesiomonas shigelloides and Aeromonas species isolated from marine mammals beached in Brazil in the period from 2003 to 2004

\begin{tabular}{|c|c|c|c|c|c|c|c|c|c|}
\hline \multirow[b]{2}{*}{ Microorganisms } & \multicolumn{9}{|c|}{ Marine Mammals } \\
\hline & $\begin{array}{c}\text { Pontoporia } \\
\text { blainvillei }\end{array}$ & $\begin{array}{c}\text { Sotalia } \\
\text { guianensis }\end{array}$ & $\begin{array}{c}\text { Otaria } \\
\text { byronia }\end{array}$ & $\begin{array}{l}\text { Stenella } \\
\text { frontalis }\end{array}$ & $\begin{array}{c}\text { Delphinus } \\
\text { sp. }\end{array}$ & $\begin{array}{c}\text { Eubalaena } \\
\text { australis }\end{array}$ & $\begin{array}{c}\text { Mesoplodo } \\
\text { mirus }\end{array}$ & $\begin{array}{l}\text { on Steno } \\
\text { bredanensis }\end{array}$ & $\begin{array}{c}\text { Total } \\
(\%)\end{array}$ \\
\hline A. veronii biogroup veronil & 15 & 2 & - & - & 1 & 2 & 1 & 1 & 19.3 \\
\hline Aeromonas sp. & 13 & - & 3 & 1 & 1 & - & - & - & 15.8 \\
\hline Aeromonas caviae & 9 & 1 & 2 & 1 & 1 & - & - & - & 12.3 \\
\hline Aeromonas hydrophila & 4 & 3 & 1 & 2 & - & 1 & - & - & 9.6 \\
\hline A. veronii biogroup sobria & 1 & 2 & 4 & 1 & 2 & - & - & - & 8.8 \\
\hline Plesiomonas shigelloides & 5 & 2 & 1 & - & - & - & - & - & 7.0 \\
\hline Aeromonas jandaei & 4 & - & 1 & 1 & - & - & - & - & 5.3 \\
\hline Aeromonas sobria & 1 & 1 & - & 1 & 1 & - & 2 & - & 5.3 \\
\hline Aeromonas eucrenophila & 3 & 1 & - & - & - & - & - & 1 & 4.4 \\
\hline Aeromonas schubertii & 4 & 1 & - & - & - & - & - & - & 4.4 \\
\hline Aeromonas media & 4 & - & 1 & - & - & - & - & - & 4.4 \\
\hline Aeromonas trota & 4 & - & - & - & - & - & - & - & 3.5 \\
\hline Total $(\%)$ & 58.8 & 11.4 & 11.4 & 6.1 & 5.3 & 2.6 & 2.6 & 2.0 & - \\
\hline
\end{tabular}


Table 3. Aeromonas and Plesiomonas shigelloides isolated from marine mammals beached or accidentally captured by fishing net in Rio de Janeiro and Rio Grande do Sul, Brazil.

\begin{tabular}{|c|c|c|c|}
\hline Marine Mammals & $\begin{array}{l}\text { Positives } \\
\text { swabs (N) }\end{array}$ & $\begin{array}{l}\text { Swabs' } \\
\text { origin }\end{array}$ & Microorganisms / $(\mathrm{N})$ \\
\hline \multirow{2}{*}{$\begin{array}{l}\text { Eubalaena australis } \\
\text { southern right whale }\end{array}$} & \multirow[t]{2}{*}{3} & Blowhole & $\begin{array}{l}\text { Aeromonas veronii biogroup veronii (1) } \\
\text { Aeromonas hydrophila (1) }\end{array}$ \\
\hline & & Anus & Aeromonas veronii biogroup veronii (1) \\
\hline \multirow{3}{*}{$\begin{array}{l}\text { Mesoplodon mirus } \\
\text { True's beaked whale }\end{array}$} & \multirow{3}{*}{3} & Genital slit & Aeromonas sobria $(1)$ \\
\hline & & Mouth & Aeromonas veronii biogroup veronii (1) \\
\hline & & Wound & Aeromonas sobria $(1)$ \\
\hline \multirow{13}{*}{$\begin{array}{l}\text { Sotalia guianensis } \\
\text { marine tucuxi dolphin }\end{array}$} & \multirow{13}{*}{8} & Blowhole & Plesiomonas shigelloides (1) \\
\hline & & & Aeromonas sobria (1) \\
\hline & & & Aeromonas hydrophila (1) \\
\hline & & & Aeromonas schubertii (1) \\
\hline & & Anus & Aeromonas veronii biogroup sobria (1) \\
\hline & & & Aeromonas hydrophila (1) \\
\hline & & Genital slit & Aeromonas veronii biogroup veronii (1) \\
\hline & & & Plesiomonas shigelloides (1) \\
\hline & & & Aeromonas hydrophila (1) \\
\hline & & & Aeromonas caviae (1) \\
\hline & & Mouth & Aeromonas eucrenophila (1) \\
\hline & & & Aeromonas veronii biogroup veronii (1) \\
\hline & & & Aeromonas veronii biogroup sobria (1) \\
\hline \multirow{6}{*}{$\begin{array}{l}\text { Stenella frontalis } \\
\text { Atlantic spotted dolphin }\end{array}$} & \multirow{6}{*}{8} & Blowhole & Aeromonas sp. (1) \\
\hline & & & Aeromonas caviae (1) \\
\hline & & Genital slit & Aeromonas hydrophila (2) \\
\hline & & Anus & Aeromonas jandaei (1) \\
\hline & & Intestine & Aeromonas sobria (1) \\
\hline & & Intestine & Aeromonas veronii biogroup sobria (1) \\
\hline \multirow{6}{*}{$\begin{array}{l}\text { Delphinus sp. } \\
\text { common dolphin }\end{array}$} & \multirow{6}{*}{4} & Blowhole & Aeromonas veronii biogroup sobria (1) \\
\hline & & & Aeromonas caviae (1) \\
\hline & & Genital slit & Aeromonas veronii biogroup sobria (1) \\
\hline & & & Aeromonas sobria $(1)$ \\
\hline & & & Aeromonas sp. (1) \\
\hline & & Anus & Aeromonas veronii biogroup veronii (1) \\
\hline \multirow{2}{*}{$\begin{array}{l}\text { Steno bredanensis } \\
\text { rough-toothed dolphin }\end{array}$} & \multirow[t]{2}{*}{1} & Blowhole & Aeromonas eucrenophila (1) \\
\hline & & & Aeromonas veronii biogroup veronii (1) \\
\hline \multirow{13}{*}{$\begin{array}{l}\text { Otaria byronia } \\
\text { South American sea lion }\end{array}$} & \multirow{13}{*}{5} & Anus & Aeromonas media $(1)$ \\
\hline & & & Aeromonas caviae (1) \\
\hline & & & Aeromonas jandaei (1) \\
\hline & & & Aeromonas veronii biogroup sobria (1) \\
\hline & & Mouth & Aeromonas veronii biogroup sobria (1) \\
\hline & & & Plesiomonas shigelloides (1) \\
\hline & & & Aeromonas sp. (1) \\
\hline & & Nostrils & Aeromonas sp. (1) \\
\hline & & & Aeromonas caviae (1) \\
\hline & & & Aeromonas veronii biogroup sobria (1) \\
\hline & & Umbilicus & Aeromonas hydrophila (1) \\
\hline & & Left eye & Aeromonas veronii biogroup sobria (1) \\
\hline & & & Aeromonas sp. (1) \\
\hline
\end{tabular}


Table 4. Aeromonas and Plesiomonas shigelloides isolated from franciscanas beached or accidentally captured by fishing net in Rio de Janeiro and Rio Grande do Sul, Brazil.

\begin{tabular}{|c|c|c|c|}
\hline Marine Mammals & $\begin{array}{l}\text { Positives } \\
\text { swabs (N) }\end{array}$ & $\begin{array}{l}\text { Swabs' } \\
\text { origin }\end{array}$ & Microorganisms / $(\mathrm{N})$ \\
\hline \multirow{5}{*}{$\begin{array}{l}\text { Pontoporia blainvillei } \\
\text { franciscana }\end{array}$} & \multirow{5}{*}{52} & Anus & $\begin{array}{l}\text { Aeromonas media }(2) \\
\text { Aeromonas veronii biogroup veronii }(1) \\
\text { Plesiomonas shigelloides }(1) \\
\text { Aeromonas sp. }(5) \\
\text { Aeromonas schubertii }(2) \\
\text { Aeromonas caviae }(3) \\
\text { Aeromonas jandaei }(2) \\
\text { Aeromonas hydrophila }(1) \\
\text { Aeromonas eucrenophila }(1)\end{array}$ \\
\hline & & Genital slit & $\begin{array}{l}\text { Aeromonas media }(1) \\
\text { Aeromonas caviae }(4) \\
\text { Aeromonas sobria }(1) \\
\text { Aeromonas veronii biogroup veronii }(4) \\
\text { Aeromonas eucrenophila }(1) \\
\quad \text { Aeromonas schubertii }(1) \\
\text { Aeromonas } \text { sp. }(2)\end{array}$ \\
\hline & & Blowhole & $\begin{array}{l}\text { Aeromonas hydrophila }(3) \\
\text { Aeromonas veronii biogroup veronii }(5) \\
\text { Aeromonas veronii biogroup sobria }(1) \\
\text { Aeromonas jandaei }(2) \\
\text { Aeromonas trota }(2) \\
\text { Aeromonas eucrenophila }(1) \\
\text { Aeromonas media }(1) \\
\text { Aeromonas sp. }(3) \\
\text { Plesiomonas shigelloides }(3)\end{array}$ \\
\hline & & Mouth & $\begin{array}{l}\text { Aeromonas trota }(2) \\
\text { Aeromonas schubertii (1) } \\
\text { Aeromonas veronii biogroup veronii }(5) \\
\text { Aeromonas caviae (2) } \\
\text { Aeromonas } \mathrm{sp}(3)\end{array}$ \\
\hline & & Right eye & Plesiomonas shigelloides (1) \\
\hline
\end{tabular}

ulcerative stomatitis and septicemia in marine mammals have also been associated with $A$. hydrophila infection $(6,18)$.

The main Aeromonas species isolated from marine mammals in this study ( $A$. veronii biogroup veronii, $A$. caviae, $A$. hydrophila, A. veronii biogroup sobria) represent important pathogens due to the possibility of causing primary or secondary septicemia and wound infections in animals and humans. The same Aeromonas species were isolated from an outbreak of diarrhea in Pernambuco, northeastern Brazil, as well as from patients with acute gastroenteritis in two hospitals at Rio Grande do Sul, southern Brazil (10,13). A similar study, developed in three municipal hospitals at Rio de Janeiro city, southeastern Brazil, showed A. caviae and A. media associated to multiresistance antimicrobial profiles as the main pathogens isolated from newborns in the Intensive Care Unit (22).

The real role of Aeromonas and $P$. shigelloides as pathogenic agents are unclear, but these microorganisms have been epidemiologically associated to acute diarrhea in human and in a diverse spectrum of diseases among both warm and coldblooded animals $(14,27)$. Probably, most of these microorganisms are not uncommon in marine mammals, but usually they are not pathogenic. However, some of these bacteria are opportunistic 
and can have the ability to cause disease or death in debilitated or immunosupressed animals. Therefore, these pathogens are important to public health due to the capacity to cause disease in humans and animals, and their monitoring is considered as a relevant tool to prevent diseases $(5,7,10,22,23)$.

Multiple infections by two or more Aeromonas species have been rarely reported (16), although it could be more common than realized. In this investigation we observed the simultaneous occurrence of different species isolated from the marine mammals. The possibility that these organisms could be acting synergistically should not be ignored $(21,27)$.

These microorganisms have a cosmopolitan distribution and are ubiquitous in the aquatic and terrestrial environments, occurring in soil, sediment, untreated and chlorinated water, human and animal infections. Considering that these bacteria are recognized as emergent pathogens $(28,30)$, we would like to emphasize the importance of this investigation which indicates the aquatic environment as a possible route of transmission among marine mammals.

Moreover this study points out the relevance of epidemiological tools and microbiological monitoring of aquatic ecosystem. This research can increase the knowledge about these microorganisms, their habitat and relationship with marine mammals and also the consequences to human health. Furthermore, these data reinforce the need to implement environmental protection programs along the Brazilian coast. The increasing degradation of marine ecosystem can have a significant impact on the health of marine mammal population, causing the emergence of new diseases.

\section{RESUMO}

\section{Plesiomonas shigelloides e patógenos da família Aeromonadaceae isolados de mamíferos marinhos da costa sul e sudeste do Brasil}

O ambiente aquático é o habitat de vários microrganismos, incluindo Plesiomonas shigelloides e espécies de Aeromonas, os quais são patogênicos para o homem e os animais. Na presente investigação, foi avaliada a ocorrência destes patógenos a partir de swabs coletados de mamíferos marinhos encalhados ou capturados acidentalmente em redes de pesca nas regiões costeiras do sudeste (RJ) e sul (RS) do Brasil. O total de 198 swabs de 27 espécimes de mamíferos marinhos, incluindo 11 espécies distintas, foi coletado por profissionais dos institutos DEENSP, GEMARS-CECLIMAR/UFRGS e enviado ao LRNCEB/IOC/FIOCRUZ. Em seguida, as amostras foram submetidas a enriquecimento em Água Peptonada Alcalina (APA) adicionada de $1 \%$ de cloreto de sódio $(\mathrm{NaCl})$ e $\mathrm{APA}$ com $3 \%$ de $\mathrm{NaCl}\left(37^{\circ} \mathrm{C} / 18-24 \mathrm{~h}\right)$. Posteriormente, as amostras foram semeadas em meio Agar Seletivo para PseudomonasAeromonas (Agar GSP) e as colônias suspeitas submetidas à caracterização bioquímica. Um total de 114 cepas foram identificadas, incluindo dez espécies de Aeromonas e $P$. shigelloides. Os principais patógenos isolados foram $A$. veronii biogrupo veronii (19,3\%), A. caviae (12,2\%), A. hydrophila $(9,6 \%)$ e Plesiomonas shigelloides (7\%). Os patógenos foram encontrados tanto em espécies de mamíferos marinhos costeiros como oceânicos. Esses dados apontam para a importância da vigilância epidemiológica e monitoramento microbiológico, além de reforçar a necessidade de implantação de programas de proteção ambiental, particularmente relacionados aos mamíferos marinhos ameaçados de extinção.

Palavras-chave: Aeromonas, Plesiomonas shigelloides, mamíferos marinhos, ecossistema aquático.

\section{REFERENCES}

1. Abbott, S.L.; Cheung, W.K.W.; Janda, J.M. (2003). The genus Aeromonas: biochemical characteristics, atypical reactions, and phenotypic identification schemes. J. Clin. Microbiol., 41: 23482357.

2. Bossart, G.D. (2006). Marine mammals as sentinel species for oceans and human health. Oceanogr., 19: 44-47.

3. Buck, J.D.; Wells, R.S.; Rhinehart, H.L.; Hansen, L.J. (2006). Aerobic microorganisms associated with free-ranging bottlenose dolphins in coastal Gulf of Mexico and Atlantic Ocean waters. J. Wildl. Dis., 42: 536-544.

4. Baker, D.A.; Smitherman, R.O.; McCaskey, T.A. (1983). Longevity of Salmonella typhimurium in Tilapia aurea and water from pools fertilized with swine waste. Appl. Environm. Microbiol., 45: 15481558.

5. Cavalcanti, A.D. (2003). Monitoramento da contaminação por elementos-traço em ostras comercializadas em Recife, Pernambuco, Brasil. Cad. Saúde Pub., 19: 1545-1551.

6. Cusick P.K.; Bullock B.C. (1973). Ulcerative stomatitis and pneumonia associated with Aeromonas hydrophila infection in the bottle-nosed dolphin. J. Am.Vet. Med. Assoc., 163: 578

7. Esposto, E.M.; Silva, W.C.P.; Reis, C.M.F.; Reis, E.M.F.; Ribeiro, R.V.; Rodrigues, D.P.; Lázaro, N.S. (2007). Enteropatógenos bacterianos em peixes criados em uma estação de reciclagem de nutrientes e no ecossistema relacionado. Pesq. Vet. Bras., 27: 144;148.

8. Evangelista, N.S.B.; Vieira, R.H.S.F.; Carvalho, F.C.T.; Torres. R.C.O.; Sant'anna, E.S.; Rodrigues, D.P.; Reis, C.M.F. (2006). Aeromonas spp. isolated from oysters (Crassostrea rhizophorae) from a natural oyster bed, Ceará, Brazil. Rev. Inst. Med. Trop. S. Paulo, 48: 129-133.

9. Food and Drug Administration (FDA). Bacteriologycal Analytical Manual. On line version http://www.cfsan.fda.gov/ ebam/bam-9.html

10. Guerra, I.M.F.; Fadanelli, R.; Figueiro, M.; Schreiner, F.; Delamare, A.P.L.; Wollheim, C.; Costa, S.O.P.; Echeverrigaray, S. (2007). Aeromonas associated diarrhoeal disease in South Brazil: prevalence, virulence factors and antimicrobial resistance. Braz. J. Microbiol., 38: 638-643.

11. González-Rey, C.; Svenson, S.B.; Bravo, L.; Siitonen, A.; Pasquale, V.; Dumontet, S.; Ciznar, I.; Krovacek, K. (2004). Serotypes and antimicrobial susceptibility of Plesiomonas shigelloides isolates from humans, animals and aquatic environments in different countries. Comp. Immunol. Microbiol. Infect. Dis., 27: 129-139.

12. Hall, A.J.; Hugunin, K.; Deaville, R.; Law, R.J.; Allchin, C.R.; Jepson, P.D. (2006). The Risk of Infection from Polychlorinated Biphenyl Exposure in the Harbor Porpoise (Phocoena phocoena): A CaseControl Approach. Environ. Health Perspect., 114 (5): 704-711 
13. Hofer, E.; Reis, C.M.F.; Theophilo, G.N.D.; Cavalcanti, V.O.; Lima, N.V.; Henriques, M.F.C.M. (2006). Envolvimento de Aeromonas em surto de doença diarréica aguda em São Bento do Una, Pernambuco. Rev. Soc. Bras. Med. Trop., 39: 217-219.

14. Janda, J.M.; Abbot, S.L. (1998). Evolving concepts regarding the genus Aeromonas: an expanding panorama of species, disease presentations and unanswered questions. Clin Infect Dis., 27: 332 334.

15. Johnson, S.; Lowenstine, L.; Gulland, R.; Jang, S.; Imai, D.; Almy, F.; DeLong, R.; Gardner, I. (2006). Aerobic bacterial flora of the vagina and prepuce of California sea lions (Zalophus Californianus) and investigation of associations with urogenital carcinoma. Vet. Microbiol., 114: 94-103.

16. Joseph, S.W.; Carnahan, A.M.; Brayton, P.R.; Fanning, G.R.; Almazan, R.; Drabick, C.; Trudo Jr., E.W.; Colwell, R.R. (1991). Aeromonas jandaei and Aeromonas veronii dual infection of a human wound following aquatic exposure. J. Clin. Microbiol., 29: 565-569.

17. Koelle, K.; Pascual, M.; Yunus, M. (2005). Pathogen adaptation to seasonal forcing and climate change. Proc. Biol. Sci., 272: 971-7.

18. Krovacek, K.; Huang K.; Sternberg, S.; Svenson, S.B. (1998). Aeromonas hydrophila septicaemia in a grey seal (Halichoerus grypus) from the Baltic Sea: a case study. Comp. Immun. Microbiol. Infect. Dis., 21: 43-49.

19. Leaper, R.; Cooke, J.; Trathan, P.; Reid, K.; Rowntree, V.; Payne, R. (2006). Global climate drives southern right whale (Eubalaena australis) population dynamics. Biol. Lett., 2: 289-292.

20. Mondino, S.S.; Nunes, M.P.; Ricciardi, I.D. (1995). Occurrence of Plesiomonas shigelloides in water environments of Rio de Janeiro city. Mem. Inst. Oswaldo Cruz, 90: 1-4.

21. Moro, E.M.P.; Weiss, R.D.N.; Friedrich, R.S.C.; Vargas, A.C.; Weiss, L.H.N.; Nunes, M.P. (1999). Aeromonas hydrophila isolated from cases of bovine seminal vesiculites in South Brazil. J. Vet. Diagn. Invest., 11: 189-191.

22. Pereira, C.S.; Amorim, S.D.; Santos, A.F.M.; Reis, C.M.F.; Theophilo, G.N.D.; Rodrigues, D.P. (2008). Caracterização de Aeromonas spp. isoladas de neonatos hospitalizados na cidade do Rio de Janeiro. Rev. Soc. Bras. Med. Trop., 41: 179-182.

23. Pereira, C.S.; Amorim, S.D.; Santos, A.F.M.; Siciliano, S.; Moreno, I.M.B.; Ott, P.H.; Rodrigues, D.P. (2007). Vibrio spp. isolados de mamíferos marinhos capturados na região litorânea do Sudeste ao Sul do Brasil. Pesq. Vet. Bras., 27: 81-83.

24. Pereira, C.S.; Possas, C.A.; Viana, C.M.; Rodrigues, D.P. (2004). Aeromonas spp. e Plesiomonas shigelloides isoladas a partir de mexilhões (Perna perna) in natura e pré-cozidos no Rio de Janeiro, RJ. Ciênc. Tecnol. Aliment., 24: 562-566.

25. Siciliano, S.; Alves, V.C.; Hacon, S. (2005). Aves e mamíferos marinhos como sentinelas ecológicas da saúde ambiental: uma revisão do conhecimento brasileiro. Cadernos Saúde Coletiva, Rio de Janeiro, 13: 927-946.

26. Simmonds, M.P.; Isaac, S. (2005). Climate change and marine apex predators: some warning signals. p. 26-33. In: Migratory Species and Climate Change: Impacts of a Changing Environment on Wild Animals. UNEP/ CMS Secretariat, Bonn, Germany. 68 p.

27. Sugita, H.; Tanaka, K.; Yoshinami, M.; Degushi, Y. (1995). Distribution of Aeromonas species in the intestinal tracts of river fish. Appl. Environ. Microbiol., 61: 4128-4130.

28. Tena, D.; González-Praetorius, A.; Simeno, C.; Pérez-Pomata, M.T.; Bisquert, J. (2004). Extraintestinal infection due to Aeromonas spp.: a review of 38 cases. Enferm. Infecc. Microbiol. Clin., 25: 235-241.

29. Van Bressem, M.F.; Waerebeek, K.V.; Reyes, J.; Felix, F.; Echegaray, M.; Siciliano, S.; Di Beneditto, A.P.; Flach, L.; Viddi, F.; Ávila, I.C.; Herrera, J.C.; Tóbon, I.C.; Bolaños-Jimenez, J.; Moreno, I.B.; Ott, P.H.; Sanino, G.P.; Castineira, E.; Montes, D.; Crespo, E.; Flores, P.A.C.; Hasse, B.; de Souza, S.M.F.M.; Laeta, M.; Fragoso, A.B. (2007). A preliminary overview of skin and skeletal diseasses and traumata in small cetaceans from South American waters. Lat. Am. J. Aquat. Mammal, 6 (1): 7-42.

30. Wells, R.S.; Rhinehart, H.L; Hansen, I.J.; Sweeney, J.C.; Townsed, F.I.; Stone, R.; Casper, D.; Scott, M.D.; Hohn, A.A.; Rowles, TK. (2004). Bottlenose dolphins as marine ecosystem sentinels: developing a health monitoring system. Ecohealth, 1: 246-254. 